\title{
Sensitivity of the Risk Factors for the Progression of Ocular Hypertension to Primary Open Angle Glaucoma
}

\author{
M. Waly \\ El Shorouk \\ Academy, High \\ Institutes of \\ Engineering, \\ Systems and \\ Biomedical \\ Engineering \\ Department.
}

\author{
Sharawy \\ Cairo \\ University, \\ Faculty of \\ Engineering, \\ Systems and \\ Biomedical \\ Engineering \\ Department
}

\author{
Ayman \\ Salah \\ Head of \\ ophthalmology \\ department, \\ Egypt air \\ hospital, Cairo, \\ Egypt
}

\author{
Khaeld \\ Wahba \\ Cairo \\ University, \\ Faculty of \\ Engineering, \\ Systems and \\ Biomedical \\ Engineering \\ Department
}

\author{
Islam \\ Ibrahem \\ Head of \\ medical retina \\ unite in Capital \\ eye center, \\ Cairo, Egypt
}

\begin{abstract}
In this article we evaluate the sensitivity of the risk factors of ocular hypertension progression in primary open angle glaucoma in order to distinguish between the three risk levels based on prediction classification models. The prediction classification models were trained and testing by using the most common risk factors from examination of 398 Egyptian patients. Standard classification trees as well as bagged classification were used. We classify the risk level into three risk levels which are high, middle and low based on the combination of the structural and functional risk factors. The classification outcomes of the trees were compared and we measured the sensitivity of each risk factor. The bagged classification has the best accuracy which is $87.7 \%$ for training datasets and $72.2 \%$ for testing datasets with area under the receiver operating characteristics curve (AUROC) 0.925 while decision tree gave $80 \%$ for training datasets and $68.7 \%$ for testing datasets with AUROC 0.868. The central cornea thickness (CCT) gave the best with average AUROC 0.946. Bagged classification tree promises to be a new and efficient approach for glaucoma classification. The CCT is very important risk factor due to its classification sensitivity.
\end{abstract}

\section{General Terms}

Pattern recognition, Decision tree, Bagging.

\section{Keywords}

Glaucoma, Ocular hypertension, Primary open angle glaucoma, risk factors.

\section{INTRODUCTION}

Glaucoma is the third leading cause of blindness worldwide [1]. Glaucoma is the name for a collection of heterogeneous diseases affecting the optic nerve. When the fluid level in the eye rises, the intraocular pressure (IOP) increases and glaucomatous damage ensues. It is classified as an optic neuropathy because it is through damage to the viability of the optic nerve that glaucoma results in visual field loss and ultimately blindness. The optic nerve, which normally sends messages from the eye to the brain and is essential for vision, is compromised and this damage often results in the loss of visual field and may progress to glaucoma [2].

A systematic review of the World Health Organization (WHO) surveys on blindness and low vision in 2002 showed that there are 37 million blind people worldwide, with $12.3 \%$
(4.4 million) attributable to glaucoma, second only to cataract $(48 \%)$ [3].

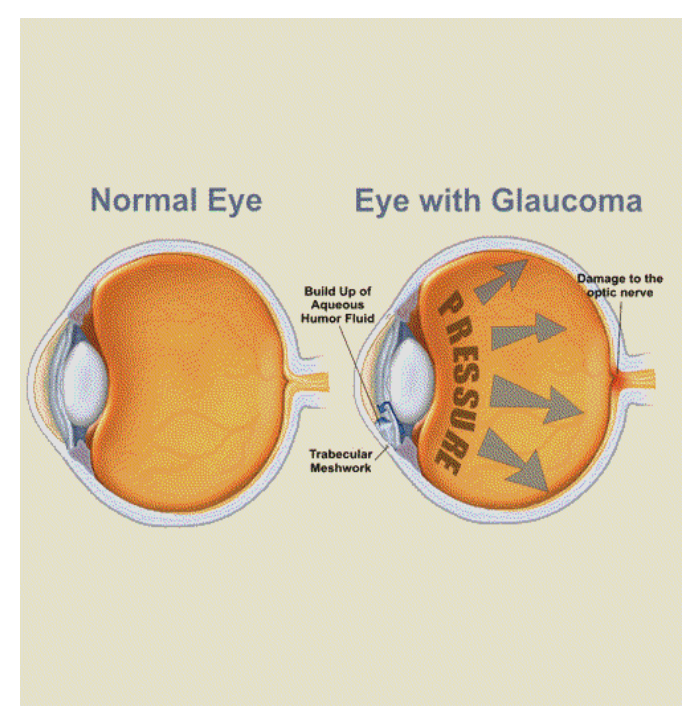

Fig1. Normal eye and eye with glaucoma

Glaucoma is called the "sneak thief" of sight because it often goes undetected in its earliest stages (Figure 1). About $50 \%$ of individuals in North America with glaucoma are not aware of it, and the problem is worse in less medically advanced portions of the world [4-5] and about $40 \%$ of the Egyptian population does not know that glaucoma causes blindness [6]. The primary source of knowledge in Egyptian population about glaucoma data for patients was mainly the ophthalmologists, followed by friends and relatives. Newspapers, TV and non-ophthalmic doctors played a smaller role, whereas radio, movies, and the Internet appeared to play no role at all (Table 1) [6].

There are several reasons why glaucoma goes undetected [7]:

- Few programs are directed towards screening exclusively for glaucoma.

- Glaucoma usually does not affect both eyes with the same degree of severity at the same time. Most patient do not know their condition as there are no clear symptoms or complaints in early stages of the diseases. 
- The progression of glaucomatous damage is usually slow. So, it is difficult for individuals to notice such gradual changes.

- Glaucoma primarily affects peripheral vision until the advanced stages of the disease process. The field of vision of the both eye complete each other.

Table 1 .Primary source of knowledge for glaucoma patients in Egypt

\begin{tabular}{|c|c|}
\hline Source of glaucoma knowledge & $\%$ \\
\hline Ophthalmologists & 79.4 \\
\hline Friends & 8.8 \\
\hline Relatives & 4.4 \\
\hline Newspapers & 2.9 \\
\hline TV & 2.9 \\
\hline Non-ophthalmic doctors & 1.5 \\
\hline Radio & 0 \\
\hline Movie & 0 \\
\hline Internet & 0 \\
\hline
\end{tabular}

There are two main types of glaucoma: primary open-angle glaucoma (POAG), in which there is no anatomical blockage to the trabecular meshwork, and angle-closure glaucoma (ACG), in which an anatomical blockage prevents access to the trabecular meshwork. To distinguish between these causes, it is necessary to perform gonioscopy to determine whether the angle is open or closed [8-10].

Primary open-angle glaucoma (POAG) is defined by open anterior chamber angles, resulting in optic nerve damage and visual field abnormalities that are unaccounted for by other diseases. A worldwide glaucoma analysis shows its devastating consequences. An extensive review of the world literature was carried out by the World Health Organization (WHO), which estimated the incidence of newly diagnosed cases of POAG at 2400000 per year [10]; POAG is a substantial public health problem as clearly demonstrated in the literature (Table 2) [10-22].

Patients affected by ocular hypertension (OHT) are usually identified on the basis of IOP $(>21 \mathrm{mmHg}$ on repeated measurements), visual field (VF) and optic disc - which should, by definition, be normal. The prevalence of OHT in those older than 40 years in the US population may vary between 4 and $7 \%$, and a number of the OHT individuals will eventually develop open angle glaucoma (OAG). For these reasons OHT has been studied extensively in order to evaluate whether it is possible to prevent or delay the development of POAG and to assess its natural history and the risk factors associated with the development of POAG.

In the current study we will seek to study the sensitivity of risk factors in order to classify Egyptian subjects having OHT which may progress to POAG into three stages based on the proposed prediction model.

Table.2 Rate of glaucoma and blindness from glaucoma

\begin{tabular}{|c|c|c|}
\hline Study & Rate of glaucoma & Rate of blindness \\
\hline Framingham Eye & $3.3 \%$ & N/A \\
\hline
\end{tabular}

\begin{tabular}{|c|c|c|}
\hline Study (1977) [11] & & \\
\hline $\begin{array}{c}\text { Baltimore Eye } \\
\text { Survey (1991) [12] }\end{array}$ & $4.7 \%$ & $13 \%$ \\
\hline $\begin{array}{l}\text { Beaver Dam Eye } \\
\text { study (1992) [13] }\end{array}$ & $2.1 \%$ & N/A \\
\hline $\begin{array}{c}\text { Proyecto Vision } \\
\text { Evaluation and } \\
\text { Research (2002) [14] }\end{array}$ & N/A & $18 \%$ \\
\hline $\begin{array}{c}\text { Olmsted County } \\
(1998,2001)[15,16]\end{array}$ & $\begin{array}{c}14.5 \text { per } 100000 \\
\text { annually }\end{array}$ & $19.3 \%$ \\
\hline $\begin{array}{c}\text { Barbados Eye Study } \\
\text { (1994) (1994) [17] }\end{array}$ & $7 \%$ & N/A \\
\hline $\begin{array}{c}\text { Prevent Blindness } \\
\text { America (2002) [18] }\end{array}$ & $4 \%$ & $10 \%$ \\
\hline St Lucia (1989) [19] & $8.8 \%$ & N/A \\
\hline $\begin{array}{c}\text { Rotterdam Study } \\
\text { (1994) [4] }\end{array}$ & $1.1 \%$ & $2.9 \%$ \\
\hline $\begin{array}{c}\text { Blue Mountains Eye } \\
\text { Study (1996) [20] }\end{array}$ & $3 \%$ & $8.8 \%$ \\
\hline $\begin{array}{l}\text { Melbourne Visual } \\
\text { Impairment } \\
\text { Project (1997) [21] }\end{array}$ & $1.7 ? \%$ & N/A \\
\hline Chen P (2003) [22] & N/A & $15 \%$ \\
\hline WHO [10] & N/A & $39 \%$ \\
\hline
\end{tabular}

\section{MATERIALS}

Making predictions is an essential part of health care. Prediction models have been continuously developed in several areas of medicine and their use has significantly contributed to the management of many disorders. The identification of risk factors for the development or progression of disease is a fundamental component in the construction of prediction models.

\subsection{What is a Risk Factor?}

In epidemiology texts, the cause of a disease is defined as an event, condition, or characteristic that plays an essential role in producing the occurrence of the disease [23]. For any example of a disease, there are a group of component causes that collectively lead to an immediate cause to produce the disease. If there is a disease in which every group of component causes always contains one particular cause, then that factor would constitute a necessary cause. For each disease, it is also important to define the effect to which the causes lead - in other words, we require a disease definition. Until recently, such a definition for POAG was largely subjective and not subject to rigorous scientific standards [24].

The term "risk factor'" describes features that may be causal in disease, as they are statistically associated with the disease, and were (or could have been) present before its occurrence, and could conceivably have played an "essential role" along with other factors in incident disease. We could call them causes, but the nature of medical and epidemiologic knowledge is such that this is probably not a good idea. We 
are, in fact, rarely going to get down to first causes in disease, so there is a good reason to stay away from causal terminology and stick with risk factor as the description [25].

The concept of risk factor is important in modern medicine, because each factor represents a potential target for a new therapy for treatment or prevention. For example, behavioral risk factors point toward behavioral changes as therapy, and genetic risk factors point toward gene therapy [25].

A few large, prospective, longitudinal studies have provided evidence with regard to the risk factors for conversion from ocular hypertension to glaucoma.

From these studies, two were randomized clinical trials, the Ocular Hypertension Treatment Study OHTS [25] and the European Glaucoma Prevention Study (EGPS ) [26]. These two studies have provided the basis for the development and validation of nowadays available prediction models for glaucoma development. Both studies have evaluated a large number of predictive factors for their potential association with the risk of conversion to glaucoma. When pooled analyses of the OHTS and EGPS data were conducted, only five baseline factors were identified as significantly associated with the risk of conversion to glaucoma [27]:

Intraocular pressure (IOP), Central corneal thickness, Age, The measurement of the vertical cup/disc ratio of the optic nerve and The visual-field pattern standard deviation (PSD)

Table 3 shows relative risks for the baseline predictive factors found to be significantly associated with the risk of developing glaucoma in these two studies. These predictive factors have been incorporated into predictive models to estimate the risk of conversion from ocular hypertension to glaucoma [27].

Below, we review some of the evidence with regard to the predictive value of risk factors reported to be associated with glaucoma development.

Table.3 OHTS vs. EGPS - risk factors

\begin{tabular}{|c|c|c|c|c|}
\hline \multirow{2}{*}{ Risk Factors } & \multicolumn{2}{|c|}{$\begin{array}{c}\text { OHTS } \\
\text { observation } \\
\mathrm{N}=819\end{array}$} & \multicolumn{2}{|c|}{$\begin{array}{c}\text { EGPS placebo } \\
\quad \mathrm{N}=522\end{array}$} \\
\hline & HR & $\begin{array}{c}95 \% \\
\text { CI }\end{array}$ & HR & $\begin{array}{c}95 \% \\
\text { CI }\end{array}$ \\
\hline Age (per decade) & 1.12 & $\begin{array}{c}0.91- \\
1.39\end{array}$ & 1.39 & $\begin{array}{l}1.01- \\
1.91\end{array}$ \\
\hline $\mathrm{IOP}($ per $\mathrm{mmHg})$ & 1.22 & $\begin{array}{l}1.12- \\
1.32\end{array}$ & 1.1 & $\begin{array}{l}0.97- \\
1.26\end{array}$ \\
\hline $\begin{array}{l}\mathrm{CCT}(\text { per } 40 \mu \mathrm{m} \\
\text { thinner) }\end{array}$ & 2.03 & $\begin{array}{l}1.61- \\
2.55\end{array}$ & 2.12 & $\begin{array}{l}1.51- \\
2.97\end{array}$ \\
\hline $\begin{array}{l}\mathrm{PSD}(\text { per } 0.2 \mathrm{~dB} \\
\text { greater })\end{array}$ & 1.19 & $\begin{array}{c}0.97- \\
1.45\end{array}$ & 1.06 & $\begin{array}{l}0.96- \\
1.17\end{array}$ \\
\hline $\begin{array}{c}\text { Vertical C/D ratio (per } \\
0.1 \text { larger) }\end{array}$ & 1.27 & $\begin{array}{l}1.14- \\
1.43\end{array}$ & 1.26 & $\begin{array}{l}1.03- \\
1.53\end{array}$ \\
\hline
\end{tabular}

\subsubsection{Intraocular pressure}

In the pooled analysis of the OHTS and EGPS control groups (1319 patients followed-up without treatment), a $1 \mathrm{mmHg}$ higher baseline IOP was associated with a 9\% higher risk of developing POAG ( Hazard Ratio HR $=1.09$; the 95\% confidence interval CI $=1.03-1.17$ ), after adjustment for other predictive factors [28]. It is important to note that even for this pooled analysis; the $95 \%$ confidence interval for the development of POAG was still relatively large, ranging from 1.03 to 1.17 . That is, each $1 \mathrm{mmHg}$ increased IOP could be associated with a $3 \%$ to $17 \%$ increased risk [29].

Because of the known association between elevated intraocular pressure (IOP) and glaucoma, IOP measurement has been considered a fundamental part of glaucoma evaluations for many years. Today, IOP remains important as a diagnostic indicator, but it is not the same critical component it used to be in the past. Lowering of IOP is unrelated to the diagnosis, although it remains the only modifiable risk factor in treatment [30].

Historical and current data have demonstrated that lowering IOP in confirmed cases of glaucoma can reduce the risk of increased damage and functional loss. Unfortunately, we now know that most untreated glaucoma patients in Asian countries have IOP levels that are considered to be within normal limits (normal- or low-tension glaucoma) [31-33]. Also, a large percentage of glaucoma patients, in general, have IOP measurements within normal limits for a single measurement [31]. Additionally, using IOP as a method of screening for glaucoma has less likelihood of diagnosing glaucoma than other clinical information such as optic disc and nerve fiber layer appearance, visual field properties, central corneal thickness, and age [34].

\subsubsection{Central corneal thickness (CCT)}

Corneal thickness is another factor that has been associated with the risk of conversion from OHT to glaucoma. IOP as assessed by applanation tonometry may be overestimated or underestimated in thick or thin corneas, respectively [35-40]. A considerable subset of patients classified as having ocular hypertension may simply have thicker than average corneas that result in overestimation of what is likely a normal, true IOP.

So, OHTS patients with thicker corneas my be at lower risk for glaucoma development. In fact, the OHT patients showed that CCT was a powerful predictor of development of primary open-angle glaucoma among ocular hypertensive eyes [25]. Eyes with CCT of $555 \mu \mathrm{m}$ or less had a threefold greater risk of developing glaucoma compared with participants who had CCT of more than $588 \mu \mathrm{m}$. A $40 \mu \mathrm{m}$ thinner cornea was associated with a $71 \%$ increase in the risk of conversion to glaucoma among OHTS patients in a multivariate model adjusting for other risk factors. Similar results were found by the EGPS, with a $40 \mu \mathrm{m}$ thinner cornea being associated with a $32 \%$ increase in the risk of conversion to glaucoma in the multivariate model [37].

Ocular hypertension (OHT) patients with thinner corneas had a higher prevalence of abnormalities on this test compared to patients with thicker corneas. This additional evidence for the association between thinner corneas and the development of glaucomatous functional and structural damage supports the importance of considering central corneal thickness in the assessment of risk for the development of glaucoma in patients with ocular hypertension. The mechanism by which CCT influences the risk of developing glaucoma has not been completely established. Although the effect of corneal thickness could potentially be attributed to an artifact of tonometric measurements, it is possible that CCT could be a marker for biomechanical and structural characteristics of ocular tissues, which may influence the risk of development of glaucomatous neuropathy. Eyes with thinner corneas could have a particular structural susceptibility that would make 
them more prone to develop glaucomatous damage. Further studies are necessary to evaluate this hypothesis [38-39].

\subsubsection{Age}

There is strong evidence that older age is an independent risk factor for the progression of ocular hypertension and glaucoma. Older age has been reported as a risk factor for the development of glaucoma in patients with ocular hypertension in multiple longitudinal studies. Several population-based studies have also found that the incidence of POAG increases with older age. In both the OHTS and the EGPS groups it was found that older patients with ocular hypertension had an increased risk of conversion to glaucoma over time [26].

\subsubsection{Cup/ disc ratio and pattern standard deviation of visual field}

The OHTS as well as the EGPS and several other longitudinal studies have found that certain indicators of structural and functional integrity at baseline are predictive factors for development of overt glaucomatous optic neuropathy or visual field defects in the future. Two indices that have consistently been associated with higher risk of developing glaucoma are the vertical cup/disc ratio and the visual-field PSD, both measured at the baseline visit. Their assessment proves to be helpful in predicting which patients are more likely to develop clinically important stages of disease in the future, and their inclusion in predictive models is justified. Both the vertical cup/disc ratio and PSD were significantly associated with the risk of developing glaucoma in the multivariate model combining OHTS and EGPS datasets. A 0.1 increase in vertical cup/disc ratio was associated with a $19 \%$ higher chance of developing glaucoma [26] .

For the PSD, when the intensity of target is a $0.2 \mathrm{~dB}$ will increase in the baseline PSD value was associated with a 13\% increase in risk.

\subsubsection{Retinal Nerve fiber Layer (RNFL)}

RNFL assessment for glaucoma diagnosis and follow-up has several distinct advantages over current diagnostic approaches. It was demonstrated over 30 years ago that RNFL defects are the earliest sign of glaucoma. Since then, numerous studies have found that RNFL defects occur prior to visual field loss [40-42]. A study by Sommer et al. [41] found that $88 \%$ of ocular hypertensives who developed glaucoma had RNFL defects at the time the visual field defect was detected with standard automated perimetry (SAP). Furthermore, $60 \%$ of these patients had RNFL defects that were present 6 years prior to visual field defect. The evaluation of the RNFL is important for glaucoma diagnosis as RNFL damage often occurs earlier than can be detected with visual fields and even before optic nerve head damage. Red-free RNFL photography has many advantages, but the subjective interpretation of the results and the practical limitations of the method limit its usefulness [42].

\subsection{DATA COLLECTION}

The combination between the structural and functional techniques for detecting glaucoma can improve detection, so practitioners must determine whether the patient has glaucoma or is at risk of developing glaucoma. The clinician must combine many aspects of information, including diagnostic test results, medical history, family history, clinical observations, prior examination findings and other related information [43-45].

The present study is based on the analysis of prospectively collected data from randomly selected healthy individuals from a defined catchment area comprising glaucoma patients presenting at the department of ophthalmology of Egypt Air hospital. The study includes about 398 study participants older than 40 years.

The sample was split into a training sample consisting of 200 patients and a test sample consisting of 198 patients. The demographic data are shown in Table 4 .

Table.4 The Demographic data

\begin{tabular}{|l|l|l|l|l|}
\hline Parameter & Min & Max & Mean & $\begin{array}{l}\text { Standard } \\
\text { deviation }\end{array}$ \\
\hline Age & 40 & 75 & 55.89 & 10.91 \\
\hline IOP & 23 & 30 & 26.695 & 1.702 \\
\hline CUP/Disc & 0.1 & 0.7 & 0.351 & 0.148 \\
\hline CCT & 480 & 629 & 577.4 & 35.435 \\
\hline PSD & 1 & 2.2 & 1.325 & 0.256 \\
\hline RNFL & -10 & -0.06 & -2.347 & 1.486 \\
\hline
\end{tabular}

The Training sample was classified into three stages based on a combination of risk factors as follows:

1- High risk

- Retinal nerve fiber layer defects

- Parapapillary changes

- IOP of $30 \mathrm{mmHg}$ or more

- IOP of $26 \mathrm{mmHg}$ or more and central cornea thickness < $555 \mu \mathrm{m}$

- Vertical Cup/disc ratio of 0.4 or more and central cornea thickness $<555 \mu \mathrm{m}$

2- Middle risk

- IOP of 24-29 mmHg without nerve fiber layer defects

- IOP of 22-25 mmHg and central cornea thickness < $555 \mu \mathrm{m}$

- Vertical Cup/disc ratio of 0.4 or more and central cornea thickness 555 - $588 \mu \mathrm{m}$

- Family history of POAG in a first degree relative.

3- Low risk

- IOP of 24-29 mmHg central cornea thickness > $588 \mu \mathrm{m}$

- Vertical Cup/disc ratio 0.4 or less central cornea thickness > $588 \mu \mathrm{m}$

Each study participant underwent a comprehensive ophthalmic evaluation including review of medical history, best corrected visual acuity, slit lamp bio-microscopy, intraocular pressure measurement with Goldmann applanation tonometry gonioscopy, dilated slit lamp fundus examination with a 90-D lens, SAP using the Swedish interactive threshold algorithm (SITA) (Humphrey field analysis II, Carl Zeiss Meditec, Inc). Study participants underwent ocular imaging with commercially available optical coherence tomography 
(Cirrus OCT, Software 4.0, CarlZeiss, Meditec, Inc) in order to measure RNFL thickness.

Exclusion criteria for both groups included unreliable visual fields, angle abnormalities on gonioscopy; any diseases other than glaucoma that could affect the visual fields, and medications known to affect visual field sensitivity. Subjects with a best-corrected visual acuity worse than 20/40, spherical equivalent outside 6.5 diopters and a cylinder correction of 3.0 diopters were excluded. Poor quality stereoscopic photographs of the optic nerve head served as an exclusion ground for the glaucoma population. A family history of glaucoma was not an exclusion criterion.

Inclusion criteria for the glaucoma category were based on optic nerve damage and not visual field defects. The classification of an eye as glaucomatous or normal was based on the consensus of masked evaluations of two independent graders of a stereoscopic disc photograph.

All photographic evaluations were accomplished using a stereoscopic viewer (Asahi Pentax Stereo Viewer II) illuminated with color corrected fluorescent lighting. Glaucomatous optic neuropathy (GON) was defined by evidence of any of the following: excavation, neuro-retinal rim thinning or notching, nerve fiber layer defects, or an asymmetry of the vertical cup/disc ratio of 0.2 . Inconsistencies between grader's evaluations were resolved through adjudication by a third evaluator. Inclusion criteria for the normal category required that the subjects have normal dilated eye examinations, open angles, and no evidence of visible GON. Normal optic discs had a cup-to-disc ratio asymmetry $<=0.2$, intact rims, and no hemorrhages, notches, excavation, or nerve fiber layer defects. Normal subjects had intraocular pressure (IOP) $<=22 \mathrm{~mm} \mathrm{Hg}$ with no history of elevated IOP. Excluded from the normal population were suspects with no GON and with IOP $\Rightarrow 23 \mathrm{~mm} \mathrm{Hg}$ on at least two occasions. These suspects are part of a separate study on classification of stratified patient populations.

\section{METHOD}

Although it can be difficult to combine the large amount of data provided by currently available glaucoma detection techniques in a meaningful way, machine learning classifiers (MLCs) can accomplish this objectively. The application of machine learning methods in medicine for automated classification is a common practice after all [45-47].

\subsection{Classifiers}

As we show previously [48], the decision tree gives the best accuracy regarding the training, as well as the testing data set, due to the nature of the input data (See table 5) .

\section{Table.5 Comparison between three classifiers}

Classification trees are unstable in the way that a small change in the training data (e.g., removal or addition of some observations) may lead to large changes in the resulting trees. Breiman [49-50] introduced a method to get a more stable procedure called "bagging" (acronym for bootstrap aggregating).

\section{Bagging was performed as follows:}

First a bootstrap sample is drawn from the original training set (i.e., take a random sample with replacement of size $\mathrm{N}$ out of the $\mathrm{N}$ observations). Due to sampling with replacement, the bootstrap sample will contain some of the observations more than one time; other observations will be left out.
Second, a tree is constructed for the bootstrap sample. This

\begin{tabular}{|c|c|c|c|}
\hline Classifier & $\begin{array}{c}\text { Accuracy In } \\
\text { training data set }\end{array}$ & $\begin{array}{c}\text { Accuracy } \\
\text { in testing } \\
\text { data set }\end{array}$ & AUROC \\
\hline DT & $80 \%$ & $68.7 \%$ & 0.868 \\
\hline $\begin{array}{c}\text { Fuzzy } \\
\text { Logic }\end{array}$ & $77 \%$ & $65.1 \%$ & 0.815 \\
\hline $\begin{array}{c}\text { Neural } \\
\text { network }\end{array}$ & $77 \%$ & $67.7 \%$ & 0.844 \\
\hline
\end{tabular}

procedure is replicated a number of times and results in numerous single trees. Each level of glaucoma risk is predicted for a new subject, if most of the trees predict that level of glaucoma for this subject. In other words, the different trees "vote" for one level.

\subsection{Sensitivity analysis}

We apply the sensitivity test in order to determine the sensitivity of each risk factor so as to distinguish between the risk levels. Sensitivity and specificity are widely used statistics to describe a diagnostic test. In particular, they are used to quantify how good and reliable a test is. Sensitivity evaluates how good the test is at detecting a positive disease. Specificity estimates how likely patients without disease can be correctly ruled out. The ROC curve is a graphic representation of the relationship between both sensitivity and specificity, and it helps to decide the optimal model. Accuracy measures how correct a diagnostic test identifies and excludes a given condition. The accuracy of a diagnostic test can be determined from sensitivity and specificity with the presence of prevalence [51]. There are several terms that are commonly used along with the description of sensitivity, specificity and accuracy. They are true positive (TP), true negative (TN), false negative (FN), and false positive (FP). If a disease is proven present in a patient, and the given diagnostic test also indicates the presence of disease, the result of the diagnostic test is considered TP. Similarly, if a disease is proven absent in a patient, and the diagnostic test suggests that the disease is absent as well, the test result is TN. Both true positive and true negative suggest a consistent result between the diagnostic test and the proven condition (also called standard of truth). However, no medical test is perfect. If the diagnostic test indicates the presence of disease in a patient who actually has no such disease, the test result is FP. Similarly, if the result of the diagnosis test suggests that the disease is absent for a patient with disease for sure, the test result is FN. Both false positive and false negative indicate that the test results are opposite to the actual condition.

$$
\begin{aligned}
& \text { Sensitivity }=\mathrm{TP} /(\mathrm{TP}+\mathrm{FN}) \\
& \text { Specificity }=\mathrm{TN} /(\mathrm{TN}+\mathrm{FP})
\end{aligned}
$$

One performance measure that we used is based on the receiver operating characteristic (ROC) curve. In general, the area under the ROC curve (AUROC) gives the probability that the output value from one randomly chosen glaucoma eye, for instance, will be greater than the output value from one randomly chosen normal eye. Given two overlapping two-dimensional curves representing the distribution of the output value for each class (e.g., glaucoma eyes and normal eyes), the sensitivity (i.e., the percentage of glaucoma eyes classified as glaucoma), and specificity (i.e., the percentage of normal eyes identified as normal) will vary as the identification threshold is moved from one end of the curve to 
the other. The graph of the sensitivity on the $y$ axis vs. specificity on the $\mathrm{x}$ axis is the ROC curve. The AUROC indicates with one number the performance of the classifier. Chance alone is equivalent to an area of 0.5 and an area of 1.0 , which is equivalent to perfect accuracy.

\section{RESULTS AND DISCUSSION}

The sample was split into a training sample consisting of 200 patients and a test sample consisting of 198 patients. A confusion matrix contains information about actual and predicted classifications done by a classification system. Performance of such systems is commonly evaluated using the data in the matrix. In the field of artificial intelligence, a confusion matrix is a specific table layout that allows visualization of the performance of an algorithm, typically a supervised learning one (in unsupervised learning it is usually called a matching matrix). Each column of the matrix represents the instances in a predicted class, while each row represents the instances in an actual class [51].

\subsection{Training Results}

\subsubsection{Decision tree}

Table 6 shows the confusion matrix with an overall accuracy $=80 \%$

Table 6. The confusion matrix of DT for training data set

\begin{tabular}{|c|c|c|c|c|}
\hline \multirow{2}{*}{} & \multirow{3}{*}{} & \multicolumn{3}{|c|}{ Predictive Values } \\
\cline { 3 - 5 } & & Low & Mid & High \\
\cline { 2 - 5 } & Low & 62 & 38 & 0 \\
\cline { 2 - 5 } & Mid & 0 & 58 & 2 \\
\cline { 2 - 5 } & High & 0 & 0 & 40 \\
\cline { 2 - 5 } & & &
\end{tabular}

\subsubsection{Bagging}

Table 7 shows the confusion matrix with an overall accuracy $=87.5 \%$

\subsection{Testing Results}

\subsubsection{Decision tree}

Table 8 shows the confusion matrix with an overall accuracy $=68.7 \%$

\subsubsection{Bagging}

Table 9 shows the confusion matrix with an overall accuracy $=72.2 \%$

Table 7. The confusion matrix of bagging for training data set

\begin{tabular}{|c|c|c|c|c|}
\hline \multirow{2}{*}{} & \multirow{2}{*}{} & \multicolumn{3}{|c|}{ Predictive Values } \\
\cline { 3 - 5 } & & Low & Mid & High \\
\cline { 3 - 5 } & & 78 & 22 & 0 \\
\cline { 2 - 5 } & Low & 0 & 59 & 1 \\
\cline { 2 - 5 } & Mid & 0 & & \\
\cline { 2 - 5 } & & & & \\
\hline
\end{tabular}

\begin{tabular}{|l|l|l|l|l|}
\hline & High & 0 & 2 & 38 \\
\hline
\end{tabular}

Table 8. The confusion matrix of DT for testing data set

\begin{tabular}{|c|c|c|c|c|}
\hline \multirow{5}{*}{ 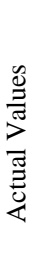 } & & \multicolumn{3}{|c|}{ Predictive Values } \\
\hline & & Low & Mid & High \\
\hline & Low & 12 & 21 & 1 \\
\hline & Mid & 0 & 39 & 34 \\
\hline & High & 0 & 6 & 85 \\
\hline
\end{tabular}

Table 9. The confusion matrix of Bagging for testing data set

\begin{tabular}{|c|c|c|c|c|}
\hline \multirow{2}{*}{} & \multirow{2}{*}{} & \multicolumn{3}{|c|}{ Predictive Values } \\
\cline { 3 - 5 } & & Low & Mid & High \\
\cline { 3 - 5 } & & 16 & 20 & 0 \\
\cline { 2 - 5 } & Low & 0 & 39 & 34 \\
\cline { 2 - 5 } & Mid & 0 & 3 & 88 \\
\cline { 2 - 5 } & High & & & \\
\hline
\end{tabular}

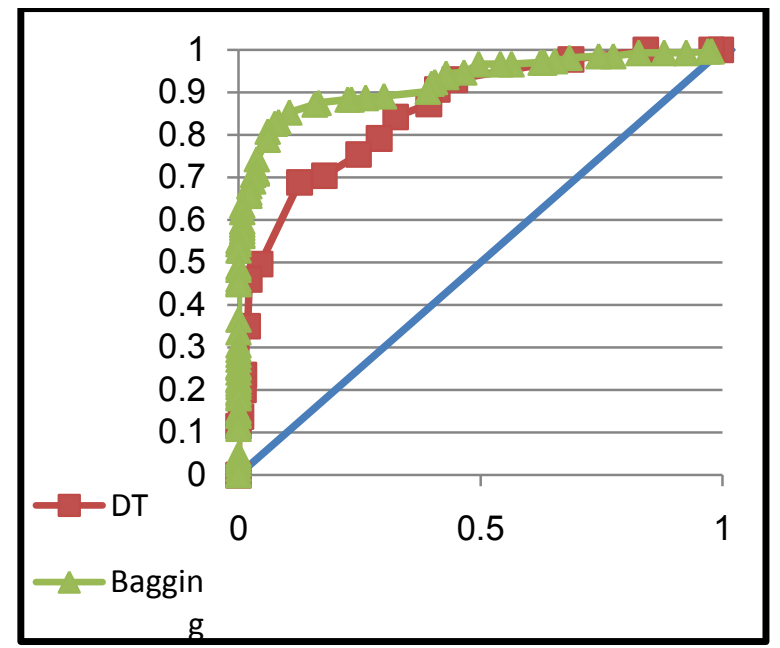

Fig2. ROC curve for Bagging and DT classifiers

Fig. 2 shows the ROC curve of DT classifer with AUROC equal to 0.868 and the ROC curve of Bagging with AUROC equal to 0.925 .

\subsection{Senstivity Resluts}

Fig. 3 shows the ROC for the risk factors for the low risk level. We notice that CCT has the biggest AUROC with 0.973; the CUP/Disc ratio has AUROC 0.946; the IOP has AUROC 0.694; the PSD has AUROC 0.608; the RNFL and Age have AUROC 0.56 . 


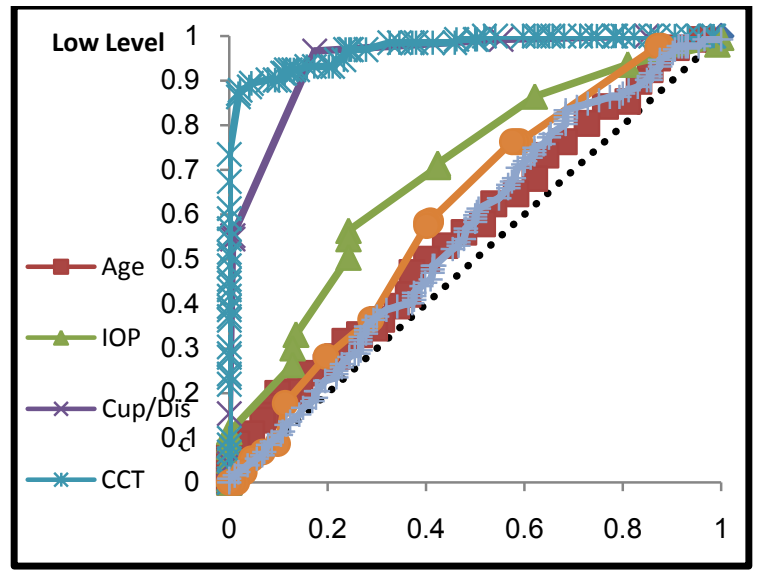

Fig3. The ROC for the risk factors during Low risk level

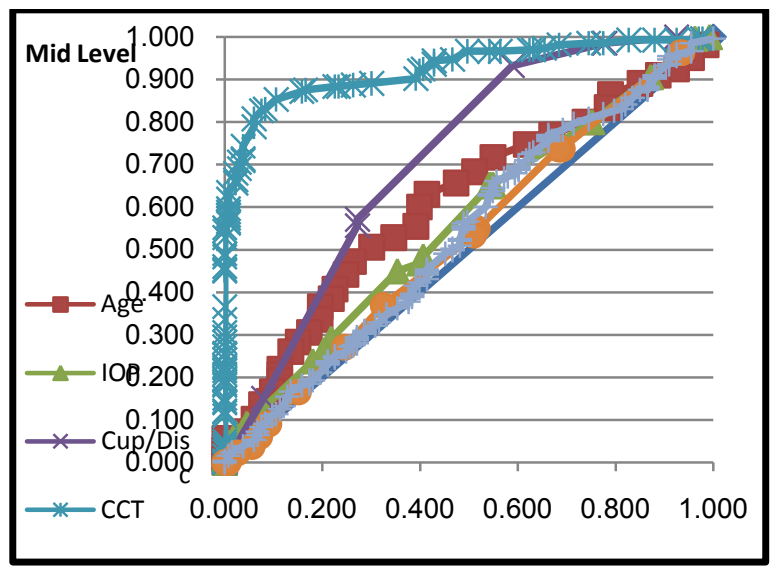

Fig4. The ROC for the risk factors during MID risk level

Fig. 4 shows the ROC for the risk factors for the middle risk level. We notice that CCT has the biggest AUROC of 0.941; the CUP/Disc ratio has an AUROC of 0.714; the IOP has an AUROC of 0.564 ; the PSD has an AUROC of 0.529; the RNFL has an AUROC of 0.573 and the age has an AUROC of 0.612 .

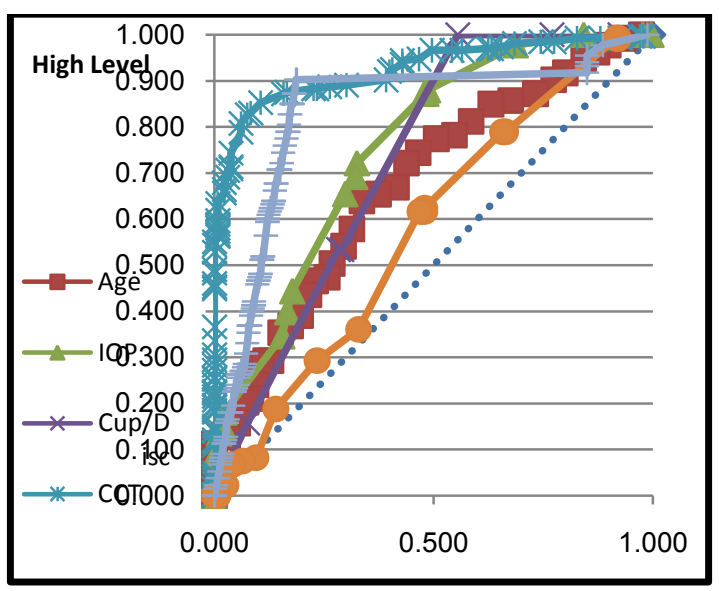

Fig5. The ROC for the risk factors during High risk level

Figure 5 shows the ROC for the risk factors during High risk level; we remark that CCT has the biggest AUROC with 0.925; the CUP/Disc ratio has AUROC 0.73; the IOP has AUROC 0.758; the PSD has AUROC 0.579; the RNFL has AUROC 0.902 and the age has AUROC 0.681 .

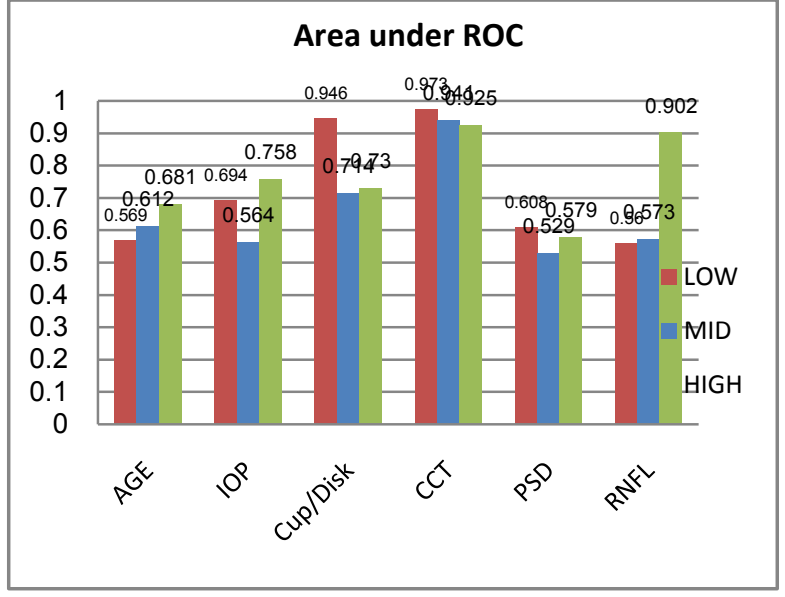

Fig6. The AUROC for each risk factor in all three risk levels

Figure 6 shows that the CCT is the best risk factors in order to identify between the three risk levels with average AUROC 0.946 then Cup/Disc ratio with average AUROC 0.796 then RNFL with average AUROC 0.678 then the IOP with average AUROC 0.672 then age 0.620 and finally PSD with average AUROC 0.572 .We can remark also that RNFL has an ability to identify the high risk patients, due to its AUROC.

\section{CONCLUSION}

Currently, the level of understanding regarding risk factors for development of glaucomatous damage and blindness from POAG is suboptimal. Identification and quantification of those factors placing the patient at greatest risk for glaucomatous damage that results in visual disability remain poorly understood. This knowledge could improve patient care and prevent loss of vision. Our goal should be not only to identify those risk factors predisposing a person to develop POAG. We should also strive to understand what factors cause progression of the disease in an individual and eventually lead to visual impairment. It is the variability in visual outcome and the inability to predict outcomes for individuals among sufferers that mandates attention to risk factors. Prospective long-term studies are needed to help identify which constellation of characteristics at diagnosis or early in its post diagnostic course are predictive of vision and quality of life outcomes. In that way we will be able to identify those patients that have increased risk of blindness and tailor treatment to improve results and prevent visual disability and blindness.

" All procedures followed were in accordance with the ethical standards of the responsible committee on human experimentation and with the Helsinki Declaration of 1975 as revised in 2000. Informed consent was obtained from all patients for being included the study "

\section{REFERENCES}

[1] Langman MJS, Lancashire RJ, Cheng K, Stewart PM. 2005. Systemic hypertension and glaucoma: mechanisms in common and co occurrence. Bri J Ophthal.; 89(8):9603.

[2] Mozaffarieh M, Grieshaber MC, Flammer J. 2008. Oxygen and blood flow: players in the pathogenesis of glaucoma. Mol Vis.; 31; 14:224-33.

[3] Bourne RR. 2006. Worldwide glaucoma through the looking glass. Br J Ophthalmol;90:253-4. 
[4] Dielemans I, VingerlingJR,Wolfs RCW, et al., 1994. The prevalence of primary open-angle glaucoma in a population-based study in the Netherlands. The Rotterdam Study, Ophthalmology; 101:1851-5.

[5] Topouzis F, Wilson MR, Harris A, et al., Prevalence of open angle glaucoma in Greece. The Thessaloniki Eye Study, Am J Ophthalmol,2007;144:511-19

[6] Mohamed Eldaly, Mostafa Hunter, Mohamed Khafagy; 2007.The socioeconomic impact among Egyptian glaucoma patients Br J Ophthalmol;91:1274-1275.

[7] G. E. Trope, Glaucoma: A Patient's Guide to the Disease (Univ. of Toronto Pr., 2001)

[8] J. J. Kanski, J. A. McAllister, 1989. Glaucoma: A Coulour Manual of Diagnosis and Treatment (Butterworths, London, Boston, Singapore, Sydney, Toronto, Wellington,

[9] Kanski JJ. 2003.CLINICAL OPHTHALMOLOGY. Oxford: Butterworth Heinemann,.(Guideline Ref ID: KANSKI2003) page 102:135.

[10] Quigley HA. 1996. Number of people with glaucoma worldwide. Br J Ophthalmol; 80:389-393.

[11] Kahn HA, Leibowitz HM, Ganley JP, et al. 1977. The Framingham Eye Study. I. Outline and major prevalence findings. Am J Epidemiol; 106:17-32.

[12] Tielsch JM, Katz J, Singh K, et al. 1991. A populationbased evaluation of glaucoma screening: the Baltimore Eye Survey. Am J Epidemiol; 134:1102-1110.

[13] Klein BE, Klein R, Sponsel WE, et al. 1992. Prevalence of glaucoma. The Beaver Dam Eye Study. Ophthalmology; 99:1499-1504.

[14] Rodriguez J, Sanchez R, Munoz B, et al. 2002. Causes of blindness and visual impairment in a population-based sample of U.S. Hispanics. Ophthalmology; 109:737-743.

[15] HattenhauerMG, Johnson DH, Ing HH, et al. 1998. The probability of blindness from open-angle glaucoma. Ophthalmology; 105:2099-2104.

[16] Schoff EO, Hattenhauer MG, Ing HH, et al. 2001. Estimated incidence of open-angle glaucoma in Olmsted County, Minnesota. Ophthalmology; 108:882-886.

[17] Leske MC, Connell AM, Schachat AP, Hyman L. 1994. The Barbados Eye Study. Prevalence of open angle glaucoma. Arch Ophthalmol; 112:821-829.

[18] National Eye Institute/Prevent Blindness America. 2002. Vision problems in the U.S.: prevalence of adult vision impairment and age-related eye disease in America. 4th ed. Chicago: Prevent Blindness America.

[19] Mason RP, Kosoko O, Wilson MR, et al. 1989. National survey of the prevalence and risk factors of glaucoma in St. Lucia, West Indies. Part I. Prevalence findings. Ophthalmology; 96:1363-1368.

[20] Mitchell P, Smith W, Attebo K, Healey PR. 1996. Prevalence of open-angle glaucoma in Australia. The Blue Mountains Eye Study. Ophthalmology; 103:16611669.
[21] Wensor MD, McCarty CA, Stanislavsky YL, et al. 1998. The prevalence of glaucoma in the Melbourne Visual Impairment Project. Ophthalmology; 105:733- 739.

[22] Chen PP. 2003.Blindness in patients with treated open angle glaucoma. Ophthalmology; 110:726-733.

[23] Rothman KJ. 1986. Modern Epidemiology. 1st ed. Boston: Little, Brown.

[24] Foster PJ, Buhrmann R, Quigley HA, et al. 2002. The definition and classification of glaucoma in prevalence surveys. Br J Ophthalmol.;86:238-242.

[25] Gordon, M.O., Beiser, J.A., et al. 2002. The ocular hypertension treatment study: baseline factors that predict the onset of primary open-angle glaucomaArch. Ophthalmol ,714-720; discussion 829-830.

[26] Miglior, S., Pfeiffer, N., et al. 2007. Predictive factors for open angle glaucoma among patients with ocular hypertension in the European glaucoma prevention study. Ophthalmology, 114(1): 3-9.

[27] Gordon, M.O., Torri, V., et al. 2007. Validated prediction model for the development of primary openangle glaucoma in individuals with ocular hypertension. Ophthalmology,,114(1): 10-19.

[28] Miglior, S. 2007. Risk factors for development of glaucoma. European Ophthalmology review „, 105(1): 4 8.

[29] Gordon, M.O. and Kass, M.A. 1999. The ocular hypertension treatment study: design and baseline description of the participants. Arch. Ophthalmol, 117(5): 573-583.

[30] Friedman DS, Wilson MR, LiebmannJM, et a. 2004. An evidence-based assessment of risk factors for the progression of ocular hypertension and glaucoma. Am J OphthalmolSep;138(3 Suppl):S19-31.

[31] Anderson DR, Drance SM, SchulzerM; 2003 Collaborative Normal-Tension Glaucoma Study Group. Factors that predict the benefit of lowering intraocular pressure in normal tension glaucoma. Am J OphthalmolNov;136(5):820-9.

[32] Shiose Y. 1984. The aging effect on intraocular pressure in an apparently normal population. Arch Ophthalmol Jun;102(6):883-7.

[33] Detry-Morel M, Zeyen T, Kestelyn P, et al. 2004. Screening for glaucoma in a general population with the non-mydriatic funds camera and the frequency doubling perimeter. Eur J Ophthalmol SepOct;14(5):387-93.

[34] Ehlers, N., Bramsen, T., et al. Applanation tonometry and central corneal thickness.ActaOphthalmol 1975,53(1): 34-43

[35] Whitacre, M.M., Stein, R.A., et al. 2004. The effect of corneal thickness on applanation tonometry. Am. J. Ophthalmol.115(5): 592-596.

[36] Herndon, L.W., Choudhri, S.A., et al. 1997. Central corneal thickness in normal, glaucomatous, and ocular 
hypertensive eyes. Arch. Ophthalmol,115(9): 11371141.

[37] Copt, R.P., Thomas, R., et al. 1999.Corneal thickness in ocular hypertension, primary open-angle glaucoma, and normal tension glaucoma. Arch. Ophthalmol,117(1): 1416.

[38] Doughty, M.J. and Zaman,M.L. 2000.Human corneal thickness and its impact on intraocular pressure measures: a review and meta-analysis approach. Surv.Ophthalmol. ,44(5): 367-408.

[39] Brandt, J.D. 2001.The influence of corneal thickness on the diagnosis and management of glaucoma. J. Glaucoma, , 10(5 (Suppl 1)): S65-S67.

[40] Sommer 1995. A. Retinal nerve fiber layer. Am J Ophthalmol.;120:665-667

[41] Sommer A, Katz J, Quigley HA, et al. 1991. Clinically detectable nerve fiber atrophy precedes the onset of glaucomatous field loss. Arch Ophthalmol.;109:77-83

[42] Sommer A, Miller NR, Pollack I, et al. 1977. The nerve fiber layer in the diagnosis of glaucoma.ArchOphthalmol.; 95:2149-2156.

[43] Caprioli j. 1992. Discrimination between normal and glaucomatous eyes.Invest opthalmolvis sci.;33;153-159
[44] Mardin CY. And Peter A. 2006. Improvingglaucoma diagnosis by combination of perimetry and HRT measurements. J Glaucoma.;15;299-305.

[45] Shah NN. 2006 .Combining structural and functional testing for detection of glaucoma. Opthalmology. $113 ; 1593-1602$.

[46] R. T. Cox, 1961. The Algebra of Probable Inference. Baltimore, MD: JohnsHopkins Univ. Press.

[47] N. Friedman, D. Geiger, and M. Goldszmidt, 1997. "Bayesian network classifiers," Machine Learning, , , vol. 29, pp. 131-163.

[48] Mohamed WALy, Amr Sahrawy and K.Wahba. 2013. A Comparison of Different Prediction Models in the Progression of Ocular hypertension to Primary Open Angle Glaucoma " International Journal of Applied Information Systems (IJAIS) -, Volume 5- No.3.

[49] Breiman L, Friedman JH, Olshen RA, et al. 1984. Classification and Regression Trees. California: Wadsworth;.

[50] Breiman L. 1998. Arcing Classifiers. The Annals of Statistics.; 26: 801-824.

[51] Stehman, Stephen V. 1997 "Selecting and interpreting measures of thematic classification accuracy". Remote Sensing of Environment. 62 (1): 77-89. doi:10.1016/S0034-4257(97)00083-7 